\title{
Analysis of grain size in FePt films fabricated using remote plasma deposition
}

DOI:

10.1016/j.jmmm.2017.07.057

\section{Document Version}

Accepted author manuscript

Link to publication record in Manchester Research Explorer

\section{Citation for published version (APA):}

Huskisson, D., Zygridou, S., Haigh, S., Barton, C., Nutter, P., \& Thomson, T. (2017). Analysis of grain size in FePt films fabricated using remote plasma deposition. Journal of Magnetism and Magnetic Materials, 443, 67-72. https://doi.org/10.1016/j.jmmm.2017.07.057

\section{Published in:}

Journal of Magnetism and Magnetic Materials

\section{Citing this paper}

Please note that where the full-text provided on Manchester Research Explorer is the Author Accepted Manuscript or Proof version this may differ from the final Published version. If citing, it is advised that you check and use the publisher's definitive version.

\section{General rights}

Copyright and moral rights for the publications made accessible in the Research Explorer are retained by the authors and/or other copyright owners and it is a condition of accessing publications that users recognise and abide by the legal requirements associated with these rights.

\section{Takedown policy}

If you believe that this document breaches copyright please refer to the University of Manchester's Takedown Procedures [http://man.ac.uk/04Y6Bo] or contact uml.scholarlycommunications@manchester.ac.uk providing relevant details, so we can investigate your claim.

\section{OPEN ACCESS}




\title{
Analysis of grain size in FePt films fabricated using remote plasma deposition
}

\author{
D. Huskisson ${ }^{1,2}$, S. Zygridou ${ }^{1}$, S. J. Haigh ${ }^{2}$, C. W. Barton ${ }^{1}$, P. W. Nutter ${ }^{1} *$ \&
}

T. Thomson ${ }^{1}$

${ }^{1}$ Nano Engineering \& Storage Technologies Research Group, The School of Computer Science, The University of Manchester, Manchester, M13 9PL, UK

${ }^{2}$ School of Materials, The University of Manchester, Manchester, M13 9PL, UK

* Corresponding author, Email: p.nutter@manchester.ac.uk

\section{Abstract}

Remote plasma sputtering (RPS) offers a high degree of control over the sputtering parameters used to deposit thin metallic films and has demonstrated a capability to control the media grain size distribution. Narrow grain size distributions remain a key requirement for future magnetic media. Here we report a comprehensive magnetometry, X-Ray diffraction and transmission electron microscopy study of how RPS affects the grain size distribution of continuous, non-segregated $\mathrm{L}_{0} \mathrm{FePt}$ thin films. These provide a model medium for heat-assisted magnetic recording and more generally for spintronic devices such as magnetoresistive random access memory and spin torque oscillators, where very high perpendicular magnetocrystalline anisotropy is required. Varying the target DC bias voltage, which in RPS can be tuned independently of the plasma generation, produces no meaningful, statistical change in average grain size, $6.5 \pm 0.1 \mathrm{~nm}$, for as-deposited, disordered FePt. Annealing at $800^{\circ} \mathrm{C}$ creates the well-ordered $\mathrm{L} 1_{0}$ phase but results in an increased average grain size of $8.3-13.6 \mathrm{~nm}$, and a significantly wider grain size distribution of $6.4-8.5 \mathrm{~nm}$. These results show that whilst RPS is capable of producing well-ordered $\mathrm{L}_{0} \mathrm{FePt}$ thin films, it does not offer an advantage in controlling the grain size of $\mathrm{FePt}$, as reported in other thin film systems.

Keywords: FePt; thin films; remote plasma sputtering; grain size distribution; x-ray diffraction; transmission electron microscopy 


\section{Introduction}

Materials with high perpendicular magnetocrystalline anisotropy (PMA) are an essential component of many rapidly developing technologies. These include spintransfer torque magnetoresistive random access memory (STT-MRAM) [1, 2], spin torque oscillators (STOs) [3] and energy assisted magnetic recording [4, 5]. High PMA materials can be used as efficient polarising layers, to bias synthetic antiferromagnetic reference layers and to maintain the thermal stability of stored data as device footprints continue to shrink.

Energy assisted magnetic recording systems will require media with high PMA together with small, well controlled grain sizes in order to meet the challenge of increased storage areal densities [5]. The grain size in magnetic storage media gives an upper bound on the storage density, since increased storage capacities require small grain sizes to maintain sufficient signal-to-noise ratio (SNR) on readout [6]. In addition, the grain size distribution must be tightly controlled to a maximum of $\pm 10 \%$ of the grain size [7] in order to allow reliable switching under the thermal/magnetic write field [8]. Ordered $\mathrm{L}_{0} \mathrm{FePt}$ thin films with PMA of $\sim 4 \times 10^{7} \mathrm{erg} / \mathrm{cm}^{3}$ are the leading contender to satisfy these requirements for heat assisted magnetic recording systems (HAMR) with storage capacities in excess of $1 \mathrm{Tbit} / \mathrm{in}^{2}[9,10]$. The high PMA found in ordered $\mathrm{L}_{0}$ FePt permits small grain sizes of $<5 \mathrm{~nm}$ whilst retaining thermal stability $[7,8]$.

Remote plasma sputtering (RPS), also known as high target utilization sputtering (HiTUS) [11], offers an alternative sputtering technique capable of finer process control than conventional magnetron systems [12, 13, 14]. Here the plasma generation is decoupled from the sputtering process so that the target DC bias voltage and acceleration of the Ar+ ions can be controlled independently of the plasma generation, allowing a greater degree of control of the sputtering process. In addition, by generating the plasma remotely and guiding it to the target material, the target is sputtered uniformly over its entire surface area. This approach is far more efficient than magnetron sputtering, where the plasma is generated locally at the target surface and sputtering is confined to a thin circular track by the magnetic field of the magnetron gun. Consequently, it also makes it a cost effective solution for the 
deposition of expensive materials such as Pt. Varying the ion impact energy, through tuning of the target DC bias voltage, has been shown to influence the average grain size in certain materials $[12,13]$, providing a method of producing thin films with controlled grain size. RPS has also been shown to allow the tuning of the anisotropy of multilayer materials through control of interfacial mixing [14]. Here, we investigate the effect on grain size and grain size distribution when non-segregated FePt films are deposited using RPS at different target DC bias voltages. Estimates of the average grain size and distribution for as-deposited and annealed FePt thin films are determined using Scherrer analysis of XRD data and through grain identification from transmission electron microscopy (TEM) images.

\section{Methods}

\subsection{Thin film Deposition \& Characterization}

FePt thin films were deposited by RPS on to $\mathrm{MgO}(001)$ substrates using a PlasmaQuest HiTUS system attached to a Lesker vacuum chamber. The films, of thickness of $10 \mathrm{~nm}$, were deposited from a $\mathrm{Fe}_{50} \mathrm{Pt}_{50}$ at\% alloy target at an $\mathrm{RF}$ power of $1 \mathrm{~kW}$ for three target DC bias voltages of $200 \mathrm{~V}, 600 \mathrm{~V}$, and $1000 \mathrm{~V}$. The chamber base pressure was better than $8 \times 10^{-9}$ mbar and an Ar pressure of $3 \times 10^{-3}$ mbar was used throughout the deposition. All sputtering was carried out at ambient temperature with no deliberate substrate heating. The as-deposited FePt films were in the expected fcc disordered phase $[15,16]$, so post annealing of the thin films was performed to induce the required $\mathrm{L} 1_{0}$ phase. The films were annealed in a conventional tube furnace at $800^{\circ} \mathrm{C}$ for one hour in an $\mathrm{Ar} / 10 \% \mathrm{H}_{2}$ reducing atmosphere to minimize oxidation. The magnetic and structural properties of the films were analyzed both before and after annealing.

Magnetic properties were measured using a MicroSense Model 10 vector vibrating sample magnetometer (VSM), which allows a (maximum) 2T magnetic field to be applied in different geometries to enable the in-plane and out-of-plane hysteresis loops to be measured, from which saturation magnetization (Ms) and coercivity (Hc) can be determined. The anisotropy field was determined through the extrapolation of the hard axis loop. All VSM measurements were performed at room temperature. 
The presence of the desired $\mathrm{L}_{0}$ ordered phase in the films was determined from XRD data produced using a Philips X'Pert Pro X-ray Diffractometer with a $\mathrm{Cu}$ anode operating at $1.2 \mathrm{~kW}$ providing $\mathrm{K}_{a}$ radiation at a wavelength of $1.5418 \AA$. The diffractometer was equipped with a Nickel filter and an X'Celerator 1D silicon strip detector. Scans were taken with a $2 \theta$ between $20^{\circ}$ and $55^{\circ}$ at $0.0334^{\circ}$ intervals with a measurement time of 80 seconds per point. Due to the instrument configuration available a large background contribution was present from the $\mathrm{MgO}$ substrate making it difficult to determine the $\mathrm{L} 1_{0}$ order parameter directly from the ratio of the (001) and (002) peaks. Consequently, ordering in the films was determined by the presence of fcc FePt (200) and $\mathrm{L}_{0} \mathrm{FePt}(001)$ and (002) peaks in the XRD data. In an ordered film we expect to observe strong, high intensity L1 ${ }_{0} \mathrm{FePt}(001)$ and (002) peaks along with a significantly reduced intensity fcc FePt (200) peak [17]. Grain sizes were estimated from the XRD data using the Scherrer equation [18, 19]. In order to determine the parameters required for the Scherrer equation, Gaussian curves were fitted to the peaks in the XRD data from which values for the full-width halfmaximum (FWHM) and peak position were determined. In the case of the disordered FePt phase the fcc FePt (200) peak was used, whereas in the case of the ordered, $\mathrm{L} 1_{0}$, phase the $\mathrm{L} 1_{0} \mathrm{FePt}(001)$ peak was used. A shape factor of 0.9 was assumed [18].

\subsection{Transmission Electron Microscopy (TEM)}

TEM was used to determine the average grain size and distribution for the asdeposited and annealed films. In order to image the samples it was necessary to reduce the thickness of the $\mathrm{MgO}$ substrate to electron transparency. Here a process of mechanical polishing using an Allied High Tech Multiprep automatic polishing machine was employed. This uses a variation on the tripod polishing technique [20], required for preserving the crystallinity of the samples, and also avoids the surface amorphization associated with ionic polishing. Samples were polished to a wedge shape orientated approximately $30^{\circ}$ to the cubic crystal lattice planes of the substrate in order to avoid fracturing and crumbling of the delicate wedge tip. The wedge had a relatively large angle of inclusion of $3^{\circ}$ in both $\mathrm{x}$ and $\mathrm{y}$ directions to further reinforce the sample. The instruments used for the TEM measurements were equipped with a $\mathrm{LaB}_{6}$ source, and an accelerating voltage of $200 \mathrm{kV}$ was used for all measurements. The as-deposited samples were imaged with a Phillips CM200 TEM and the annealed 
samples with a FEI Tecnai T20 TEM. Selected area electron diffraction (SAED) patterns were produced to identify key material features. The samples were tilted a few degrees off-axis to eliminate diffraction contrast from the $\mathrm{MgO}$ substrate. Grains were identified from dark field TEM images using the machine-learning algorithm Trainable Weka Segmentation in ImageJ [21], which provides a semi-automatic, objective, reproducible method for consistently analyzing all images using the same classifier. Feret's diameter [22] was used as a convenient measure of the grain size, and a minimum area threshold of $7 \mathrm{~nm}^{2}$ was applied to reduce false positives. Histograms of the grain size distribution were produced and fitted with lognormal distributions. The mean grain size and a measure of the width of the grain size distribution were calculated from the fitted parameters. Uncertainties in the fitting process, specifically from the difference between the data and the ideal model, are larger than the standard error of each distribution.

\section{Results and discussion}

The magnetic and structural properties of the as-deposited and annealed films were analyzed using VSM, XRD and TEM. The magnetic properties were found from hysteresis loop measurements using the VSM. Chemical ordering in the films was determined from the XRD data and both XRD and TEM were used to estimate the average grain size and, in the case of TEM, the grain size distribution.

\subsection{Magnetic and X-Ray characterization}

Figure 1 (a) shows the in-plane and out-of-plane hysteresis loops for the as-deposited, and Figure 1(b) shows the hysteresis loops after annealing, for the film deposited using a target DC bias voltage of 600V. Similar results (not shown) were observed for the other films. Figure 1(c) shows the conventional $\theta-2 \theta$ XRD data where the scattering vector is perpendicular to the plane of the film for the as-deposited and annealed films, with the key angles representing the XRD peaks of interest identified. 

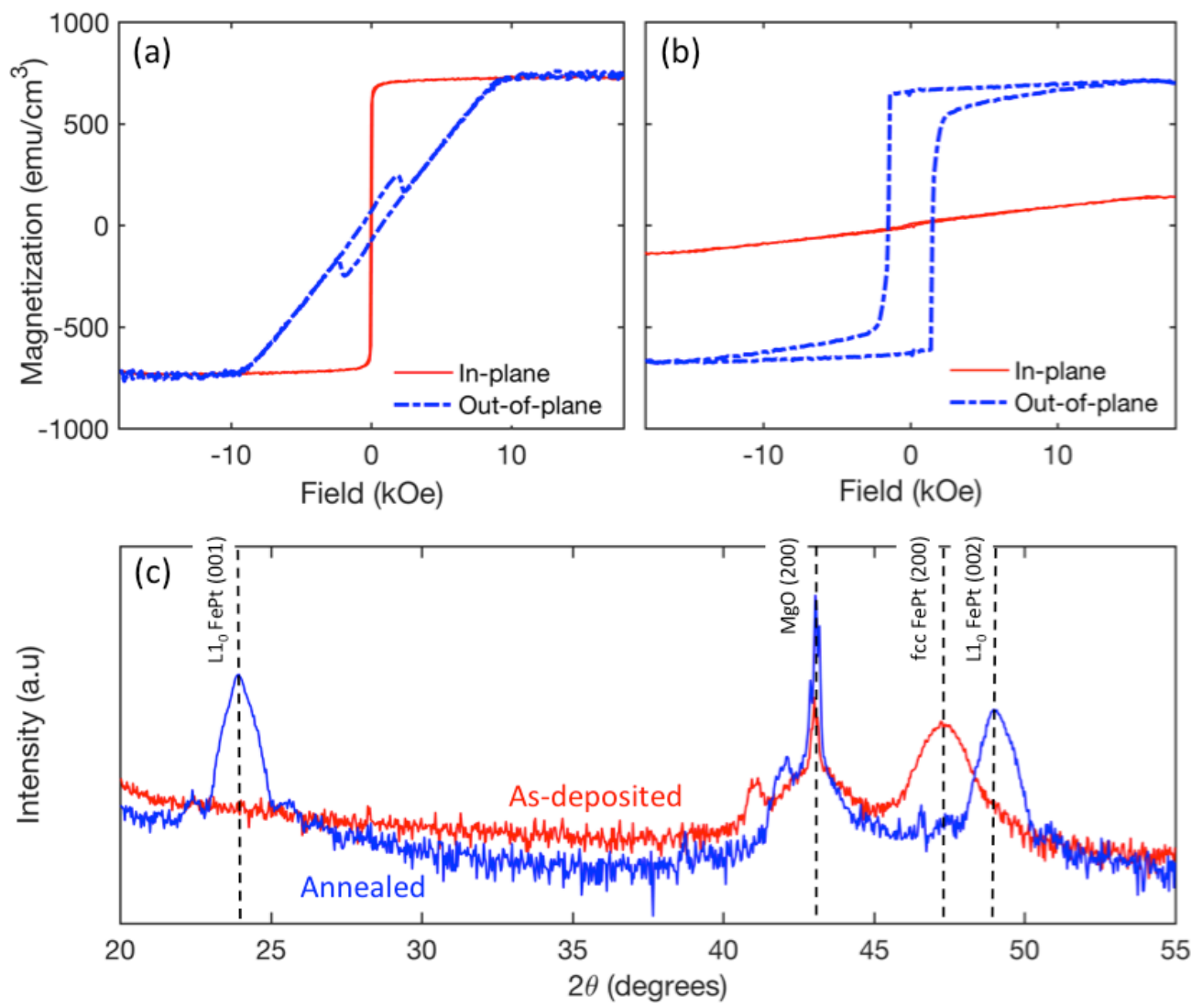

Figure 1: In-plane and out-of-plane hysteresis loops for the (a) as-deposited and (b) following annealing at $800^{\circ} \mathrm{C}$, for the $\mathrm{FePt}$ thin film deposited at a target $\mathrm{DC}$ bias voltage of $600 \mathrm{~V}$. (c) shows the conventional $\theta-2 \theta$ XRD data for the as-deposited and annealed FePt thin film deposited at a target DC bias voltage of $600 \mathrm{~V}$.

The hysteresis loops for the as-deposited films exhibit an in-plane anisotropy, which is expected for FePt thin films in the disordered fcc phase. This is confirmed by the presence of a strong fcc FePt (200) peak and the absence of $\mathrm{L1}_{0} \mathrm{FePt}(001)$ and (002) peaks in the XRD data. Following annealing, the hysteresis loops show that a strong PMA has developed as explained by the XRD data which shows high intensity $\mathrm{L}_{0}$ FePt (001) and (002) peaks and no fcc FePt (200) peak, indicating that we have developed the desired $\mathrm{L} 1_{0}$ phase following annealing. The saturation magnetization of $\mathrm{M}_{\mathrm{s}} \approx 700 \mathrm{emu} / \mathrm{cm}^{3}$ for the annealed film is lower than might be expected for bulk FePt, but a reduced $\mathrm{M}_{\mathrm{s}}$ is commonly observed in thin films [23]. Following annealing, all films had an anisotropy field $>40 \mathrm{kOe}$ as is clearly evident for the $600 \mathrm{~V}$ sample from Figure 1 (b). However, the large uncertainties in extrapolating the hard axis data preclude making a reasonable estimate of the actual value of the anisotropy field. 


\subsection{Grain size analysis}

In the case of XRD data the average height of the crystallites, and hence average grain size, can be calculated using Scherrer analysis. The average grain sizes for the asdeposited and annealed films determined using Scherrer analysis are shown in Figure 2(a). Although the films were nominally $10 \mathrm{~nm}$ thick, the calculated grain size varies between $8.3 \mathrm{~nm}$ and $10.1 \mathrm{~nm}$ for the annealed film, which is mostly smaller than the assumed films thickness. As such thin films exhibit only single grains in the out-ofplane direction [24], this suggests the presence of disordered material, maybe due to oxidation of the top surface in atmosphere after annealing, and/or oxygen migration from the $\mathrm{MgO}$ substrate during annealing. The thickness of the well ordered magnetic material within the film is therefore potentially less than the total film thickness, which could account for a lower than expected measured film magnetization. For our thin film geometry XRD is a surface reflectivity technique and is only sensitive to dimensions in the out-of-plane direction, hence, TEM was used as a direct measurement of lateral grain size, which can be compared against grain size estimates produced from Scherrer analysis of the XRD data. Representative dark field images were taken of each sample for grain size analysis, shown in Figure 3 (a) and (c) for the case of the film deposited using a target DC bias voltage of $600 \mathrm{~V}$. The SAED patterns, Figure 3 (b) and (d), evidence the good crystalline quality of the samples. The emergence of the highlighted (100) spots after annealing in Figure 3 (d) show a clear epitaxial relationship with the cubic crystal $\mathrm{MgO}$ substrate, and confirm the presence of the desired $\mathrm{L} 1_{0}$ crystal structure in the FePt demonstrated in the XRD data. Double diffraction spots are visible due to the bi-layer crystalline structure of the sample where the $\mathrm{MgO}$ substrate and the FePt thin film have slightly different lattice parameters. The TEM images reveal a preferential direction for the annealed samples, where grains of FePt can be seen to form lines, visible left to right across in Figure 3 (c), which is attributed to terracing formed by the $\mathrm{MgO}$ cut angle always present in single crystal substrates [25]. 

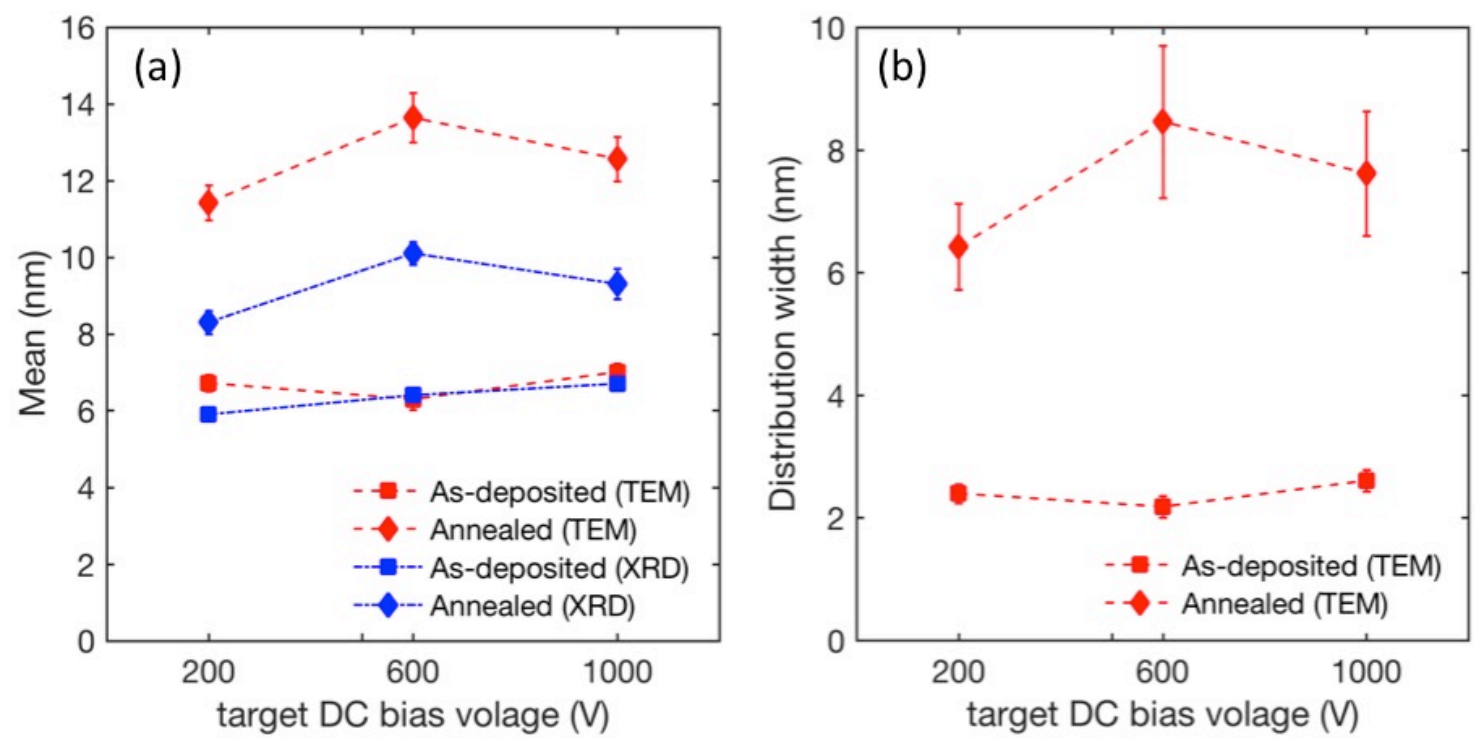

Figure 2: (a) Average grain size as a function of target DC bias voltage calculated using Scherrer analysis of the XRD peaks (dash-dot) and TEM analysis (dashed) for the as-deposited (squares) and annealed (diamonds) thin films. (b) the width of the grain size distribution determined from TEM analysis for the as-deposited (squares) and annealed films (diamonds).
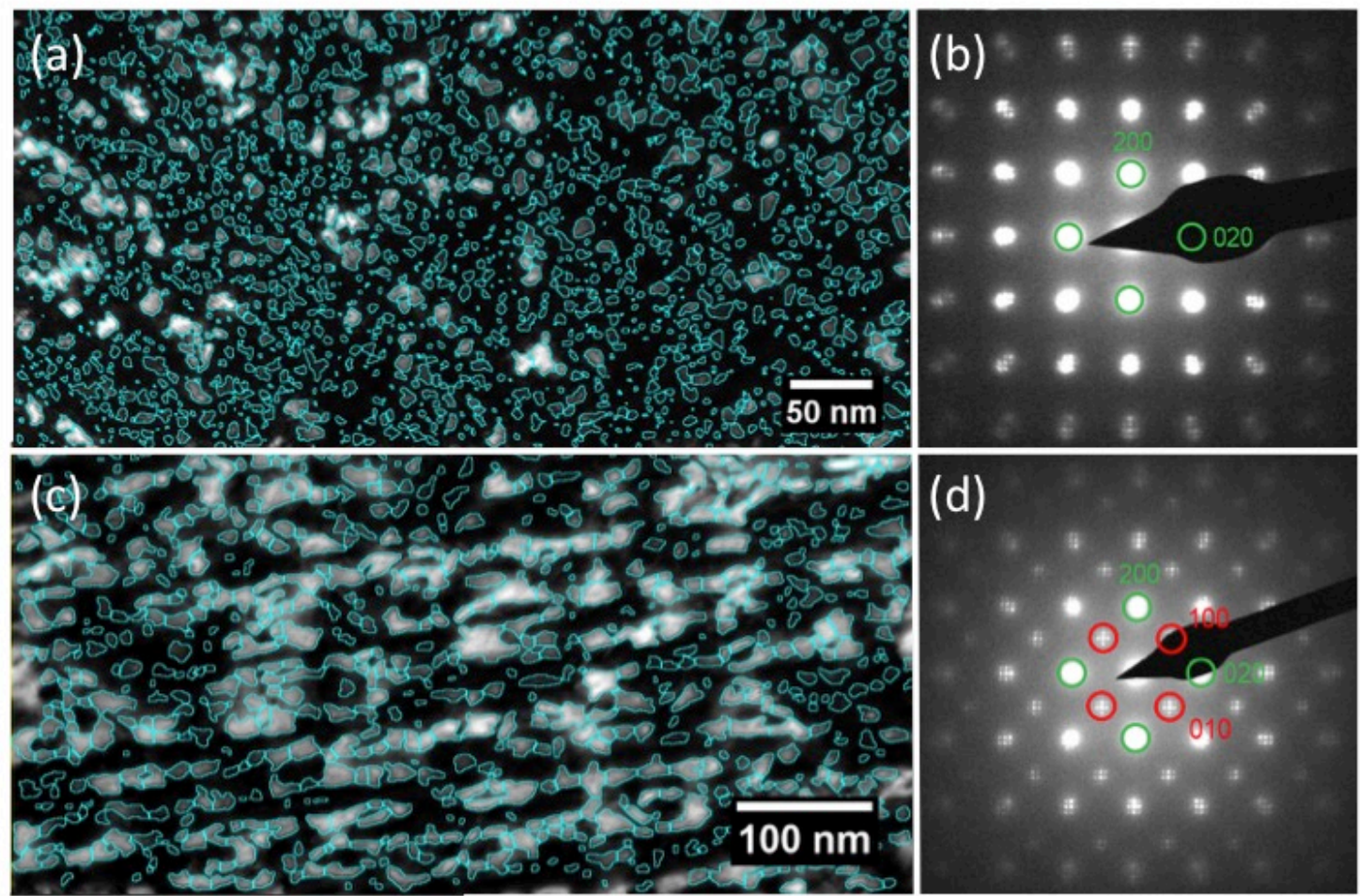

Figure 3: Representative TEM Images for the (a) as-deposited and (c) annealed, along with SAED patterns for the (b) as-deposited and (d) annealed, for the FePt thin film deposited at a target DC bias voltage of $600 \mathrm{~V}$ 
From the dark field TEM images the grains were identified and outlined using the segmentation algorithm (see Figure 3 (a) and (c)), from which a normalized probability distribution of grain sizes was determined, as shown in Figure 4, for the as-deposited and annealed films at the three target DC bias voltages. A lognormal curve was fitted to each distribution and the average grain size and width of the grain size distribution were determined from the fitted curve.
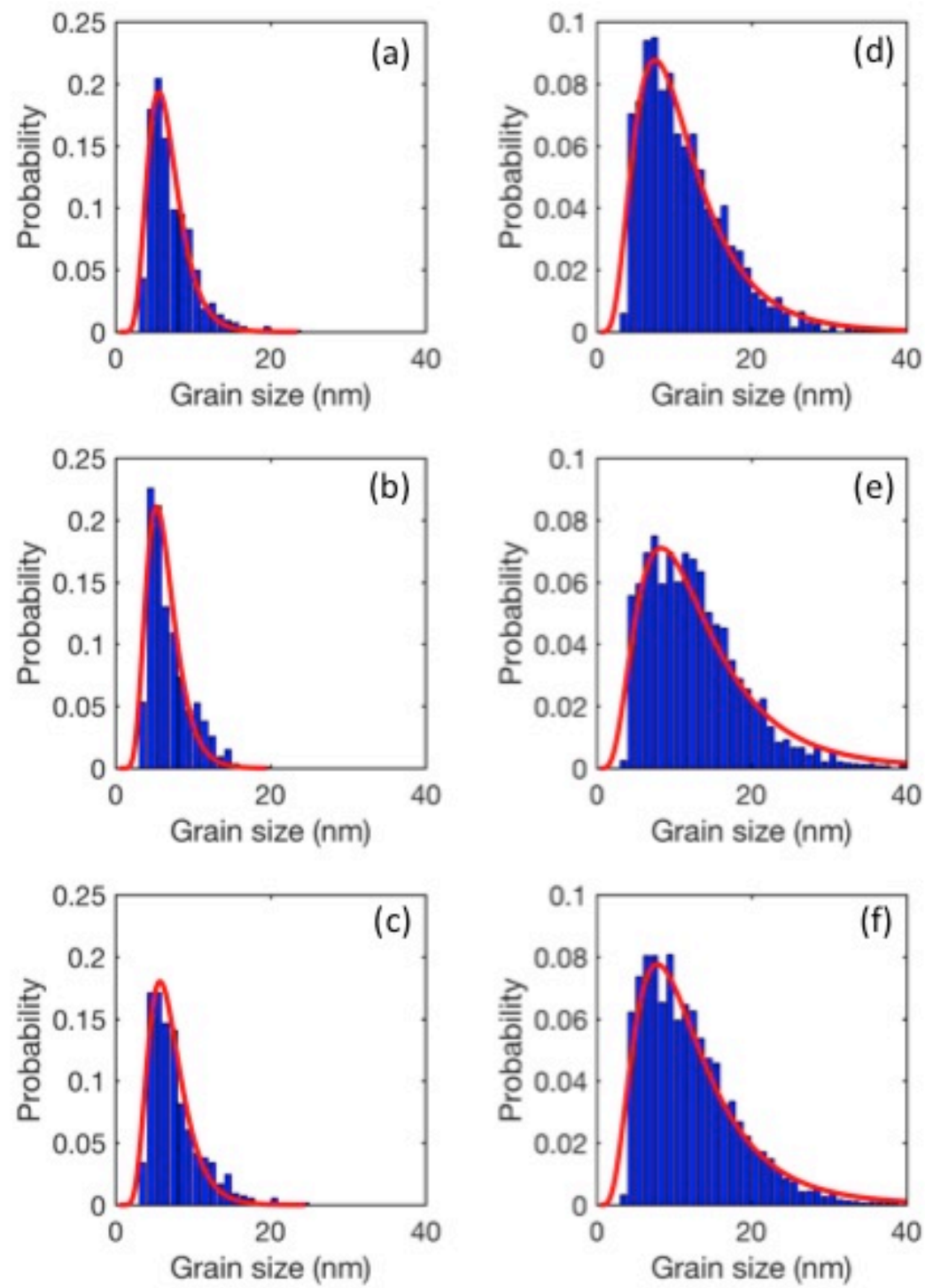

Figure 4: Grain size distributions for as-deposited (a)-(c) and annealed (d)-(f) thin films for target DC bias voltages of 200V (a) and (d), 600V (b) and (e), and 1000V (c) and (f). Solid line indicates the best-fit curve used to extract the average grain size and width of the grain size distribution. 
The calculated average grain sizes are shown in Figure 2 (a) and the width of the distribution in Figure 2 (b) for the as-deposited and annealed films. There is little difference in the average grain size calculated from the XRD data and measured from the TEM image in the as-deposited case. Both approaches predict small grain sizes with an XRD average of $6.3 \pm 0.1 \mathrm{~nm}$ (for the vertical grain size as determined from Scherrer analysis) and a TEM average of $6.7 \pm 0.2 \mathrm{~nm}$ (for the lateral grain size). For the as-deposited films equiaxed grains can be expected, and the good agreement between TEM and XRD suggests that for these films both techniques give an accurate measurement of grain size in the material. The TEM analysis further allows the grain size distribution to be determined, which is quite large with an average width of $2.4 \pm$ $0.1 \mathrm{~nm}$.

After annealing, both approaches show a significant increase in the grain size at all target DC bias voltages suggesting that the grains have grown both vertically and laterally. The TEM analysis measured a much larger increase in lateral grain size compared to XRD Scherrer analysis of the vertical grain size. The XRD average vertical grain size varied between $8.3 \pm 0.3 \mathrm{~nm}$ and $10.1 \pm 0.3 \mathrm{~nm}$, whilst the TEM average lateral grain size varied between $11.4 \pm 0.5 \mathrm{~nm}$ and $13.6 \pm 0.6 \mathrm{~nm}$. Grain growth during annealing is limited in the vertical direction by the thickness of the film, whereas the lateral grain size is less constrained. No systematic behavior was observed for different DC bias voltages, with small differences attributed to film-tofilm variation. In addition, the width of the grain size distribution is seen to increase by over a factor of three after annealing, reaching values between $6.4 \pm 0.7 \mathrm{~nm}$ and 8.5 $\pm 1.2 \mathrm{~nm}$.

The average grain size for the as-deposited films show no dependency on the target DC bias voltage. However, the greatest increase in average grain size after annealing is for a target DC bias voltage of $600 \mathrm{~V}$, with the smallest average grain size being observed at a low target DC bias voltage of $200 \mathrm{~V}$.

\section{Conclusion}

RPS has been investigated as a method of controlling the grain size of nonsegregated, prototype FePt thin films. The additional parameter space this technique 
offers has been explored to investigate possible advantages for a variety of applications. Surprisingly, considering the advantages demonstrated in other materials, there is no evidence that the grain size in FePt can be controlled by depositing using RPS. Our results demonstrate a lack of correlation between DC target voltage and grain size, indicating that the properties of this media are robust to such variation of fabrication conditions. In the as-deposited, non-ordered state the combined average grain size is shown to be $6.5 \pm 0.1 \mathrm{~nm}$, but the films exhibit a large grain size distribution of $2.4 \pm 0.2 \mathrm{~nm}$ on average. Annealing at high temperature $\left(800^{\circ} \mathrm{C}\right)$ results in the creation of the desired $\mathrm{L} 1_{0}$ phase. However, whilst well ordered films with high PMA can be produced using RPS, the resulting grains are large, varying between 8.3 and $13.6 \mathrm{~nm}$ depending on the target DC bias voltage used. More significantly, a major increase in the width of the grain size distribution is observed from $2.2 \mathrm{~nm}$ to $8.5 \mathrm{~nm}$, or $35 \%$ to $63 \%$ of the measured average grain size, for the thin film deposited at a target DC bias voltage of $600 \mathrm{~V}$. We conclude that whilst RPS offers a route to producing ordered $\mathrm{L} 1_{0} \mathrm{FePt}$ thin films, it does not offer an advantage in controlling the grain sizes and producing small, controlled grain size distributions in such thin film materials.

\section{Acknowledgements}

The authors gratefully acknowledge the support of the UK EPSRC through grant numbers EP/K008412/1 and EP/K008501/1. D.H. thanks the NOWNANO EPSRC Centre for Doctoral Training for support through grant number EP/G017905/1. T.T. and S.Z. acknowledge the contribution to funding of this work under the EU Marie Curie programme FP7-PEOPLE-2007-4-3-IRG “ANDIST”. 


\section{References}

[1] Shen J., Shi M., Tanaka T., and Matsuyama K., "Asymmetric composite free layers with compensated magnetization for ultrahigh density integration of STTMRAM”, IEEE Trans Magn, 50 (2014) 3401205.

[2] Zhou Y., Zha C.L., Bonetti S., Persson J., and Åkerman J., "Spin-torque oscillator with tilted fixed layer magnetization”, Appl Phys Lett, 92 (2008) 262508.

[3] Zhao H., Li X., Zhang Z., Ma B., and Jin Q.Y., "Study of spin valves with $\mathrm{L1}_{0^{-}}$ FePt pinning layer and different pinned layers”, IEEE Trans Magn, 43 (2007) 2839 2841.

[4] Chu H.G., Guo H.H., Xie H.L., Ma B., Zhang Z.Z., Wang Y., and Jin Q.Y., "Magnetic properties of perpendicular exchange coupled composite with a synthesis antiferromagnetic sandwich structure”, Appl Phys Lett, 104 (2014) 102402.

[5] Challener W.A., Peng C., Itagi A.V., Karns D., Peng W., Peng Y., Yang X., Zhu X., Gokemeijer N.J., Hsia Y-T., Ju G., Rottmayer R.E., Seigler M.A., and Gage E.C., "Heat-assisted magnetic recording by a near-field transducer with efficient optical energy transfer", Nature Photonics, 3 (2009) 220-224.

[6] Richter H.J., "The transition from longitudinal to perpendicular recording', J Phys D: Appl Phys, 40 (2007) R149-R177.

[7] Zhang L., Takahashi Y.K., Hono K., Stipe B.C., Juang J-Y., and Grobis M. "L10ordered $\mathrm{FePtAg}-\mathrm{C}$ granular thin film for thermally assisted magnetic recording media”, J Appl Phys, 109 (2011) 07B703.

[8] Wang J., Hata S., Takahashi Y.K., Sepehri-Amin H., Varaprasad B.S.D.Ch.S, Shiroyama T., Schrefl T., and Hono K., "Effect of MgO underlayer misoroentation on the texture and magnetic property of FePt-C granular film", Acta Mater, 91 (2015) 41-49.

[9] Varaprasad B.S.D.Ch.S., Chen M., Takahashi Y.K., and Hono K., "L110-Ordered FePt-based perpendicular magnetic recording media for heat-assisted magnetic recording”, IEEE Trans Magn, 42 (2013) 718-722.

[10] Pisana S., Mosendz O., Parker G.J., Reiner J.W., Santos T.S., McCallum A.T., and Richter H.J., "Effects of grain microstructure on magnetic properties in FePtAg-C media for heat assisted magnetic recording”, J Appl Phys, 113 (2013) 043910.

[11] Thwaites M.J., "High density plasmas” USA Patent No. 6463873, 15 Oct. 2002 [www.plasmaquest.co.uk] 
[12] Vopsaroiu M., Thwaites M.J., Fernandez G.V. Lepadatu S. and O’Grady K.,

"Grain size effects in metallic thin films prepared using a new sputtering techniques", J Optoelectron Adv M, 7 (2005) 2713-2720.

[13] Vopsaroiu M., Vallejo Fernandez G., Thwaites M.J., Anguita J., Grundy P.J., and O'Grady K. "Deposition of polycrystalline thin films with controlled grain size", J Phys D: Appl Phys, 38 (2005) 490 - 496.

[14] Barton C.W. and Thomson T., "Magnetization reversal in anisotropy graded Co/Pd multiplayers", J Appl Phys, 118 (2015) 063901.

[15] Zygridou S., Barton C.W., Nutter P.W., and Thomson T., "Exploring the potential of remote plasma sputtering for the production of $\mathrm{L}_{0}$ ordered $\mathrm{FePt}$ thin films", J Phys D: Appl Phys 50 (2017) 275005.

[16] Hsu Y-N., Jeong A., and Laughlin D.E., "Effects of Ag underlayers on the microstructure and magnetic properties of epitaxial FePt thin films”, J Appl Phys, 89 (2001) $7068-7070$.

[17] Ho H., Laughlin D.E., and Zhu J-G., “An X-ray diffraction-based method for evaluating inhomogeneous ordering at the grain level of $\mathrm{L} 1_{0}-\mathrm{FePt}$ media”, IEEE Trans Mag, 48 (2012) 2749-2752.

[18] Warren B.E., X-Ray Diffraction, Dover Publications, New York, 1990.

[19] Albrecht M. and Brombacher C., "Rapid thermal annealing of FePt thin films", Phys Status Solidi A, 210 (2013) 1272-1281.

[20] Eberg, E., Monsen, Å. F., Tybell, T., van Helvoort, A. T. J., and Holmestad, R., "Comparison of TEM specimen preparation of perovskite thin films by tripod polishing and conventional ion milling”, J Electron Microsc (Tokyo), 57 (2008) 175179.

[21] Arganda-Carreras, I.; Kaynig, V. \& Rueden, C., Eliceiri K.W., Schindelin J., Cardona A., and Seung S.S., "Trainable Weka Segmentation: a machine learning tool for microscopy pixel classification.”, Bioinformatics (2017)

https://doi.org/10.1093/bioinformatics/btx180

[22] Merkus H.G., Particle Size Measurements: Fundamental, Practice, Quality, Springer Science \& Business Media, 2009.

[23] Weller D., Mosendz O., Parker G., Pisana S., and Santos T.S., "L1 0 FePtX-Y media for heat-assisted magnetic recording”, Phys Status Solidi A, 210 (2013) 12451260. 
[24] Dong, K.F., Deng, J.Y., Peng, Y.G., Ju, G., Chow, G.M. and Chen, J.S., “Columnar structured FePt film epitaxially grown on large lattice mismatched intermediate layer", Scientific Reports 6 (2016) 34637.

[25] Sum, R., Lang, H.P., Guntherodt, H.-J., "Scanning force microscopy study of single-crystal substrates used for thin-film growth of high-temperature superconductors", Physica C, 242 (1995) 174-182. 